\title{
The jet of the Low Luminosity AGN of M81 Evidence of Precession
}

\author{
A. Alberdi ${ }^{1, a}$, I. Martí-Vidal' ${ }^{2, b}$, J.M. Marcaide ${ }^{3}$, J.C. Guirado ${ }^{3,4}$, M.A. Pérez-Torres ${ }^{1}$, E. Ros ${ }^{3,4,5}$, and A. Brunthaler ${ }^{5}$ \\ ${ }^{1}$ Instituto de Astrofísica de Andalucía (IAA-CSIC), P.O. Box 3004, 18008-Granada, Spain \\ ${ }^{2}$ ALMA Regional Center (ARC), Onsala Space Observatory, SE-439 92 Onsala, Sweden \\ ${ }^{3}$ Departament d'Astronomia i Astrofísica, Universitat de València, C. Dr. Moliner 50, E-46100 Burjassot, València, Spain \\ ${ }^{4}$ Observatori Astronòmic, Universitat de València, Parc Científic, C. Catedrático José Beltrán 2, E-46980 Paterna, València, Spain \\ ${ }^{5}$ Max-Planck-Institut für Radioastronomie, Auf dem Hügel 69, D-53121 Bonn, Germany
}

\begin{abstract}
In this contribution, we summarize our main results of a big campaign of global VLBI observations of the AGN in M81 (M81*) phase-referenced to the radio supernova SN 1993J. Thanks to the precise multi-epoch and multi-frequency astrometry, we have determined the normalized core-shift of the relativistic jet of M81* and estimated both the magnetic field and the particle density at the jet base. We have also found evidence of jet precession in M81* coming from the systematic time evolution of the jet orientation correlated with changes in the overall flux density.
\end{abstract}

\section{Introduction}

The low luminosity AGN in the galaxy M81 (hereafter, M81*), located at a distance of $3.63 \pm 0.34 \mathrm{Mpc}$ (Freedman et al. 1994[2]), has a radio luminosity of $10^{37} \mathrm{erg} / \mathrm{s}$ [3] and an X-ray luminosity of $10^{40} \mathrm{erg} / \mathrm{s}$ [14]. The mass of the central black hole has been determined by HST spectroscopic observations to be of $7 \times 10^{7} \mathrm{M}_{\odot}$ [1]. During the last fifteen years, M81* has been observed routinely, at frequencies from 1.6 to $8.4 \mathrm{GHz}$, as a phase-reference source for our monitoring of the supernova SN 1993J. M81* shows a core-jet structure (see Fig. 1), with the core region being slightly resolved at all frequencies and with a core size that increases with increasing wavelength. At each frequency, there is a jet region with inverted spectrum around its intensity peak; on the other hand, the spectral index becomes steeper as the distance from the jet base increases. All these images constitute an excellente database to study in great detail the sub-parsec scale relativistic jet in M81* (at the distance of M81, 1 milliarcsecond corresponds to $18 \mathrm{mpc}$, which is of the order of 3000 Schwarzschild Radii for the reported Black Hole mass). A recent image of $\mathrm{M} 81^{*}$ at $43 \mathrm{GHz}[15]$ has estimated a minimum size for its core of 138 Astronomical Units ( 100 Schwarzschild Radii). Combining this result with earlier measurements at longer wavelengths, Ros et al. (2012)[15] determined a frequency-size dependence of $\theta \propto v^{(-0.88 \pm 0.04)}$.

\footnotetext{
ae-mail: antxon@iaa.es

be-mail: ivan.marti-vidal@chalmers.se
}

\section{The physics of the $M 81^{*}$ jet}

It is well known that the position of the AGN jet core depends on the frequency (the "core shift" effect [6]). In fact, the accurate determination of the position shifts of the core at different frequencies provides unique information about the physical conditions of the jet (both the particle density and the magnetic field decrease with the distance to the jet base). In M81* we can study the "core shift" effect in a very robust way, since we have the advantage that SN 1993J -the phase reference source- shows an optically thin shell structure (see Fig. 1) and its central position does not depend on the frequency, favouring an absolute astrometric analysis. The SN 1993J shell is expanding spherically, with deviations from circularity $\leq 2 \%$ level, showing a self-similar expansion in a constant wind circumstellar medium [8-10].

We have performed an absolute astrometric analysis of the global VLBI observations spanning around 12 years. The first result is that the proper motion of M $81 *$ is consistent with zero in the plane of the sky. Additionally, we have determined the core-shifts for the M81* brightness peaks at 1.7, 2.3 and $5.0 \mathrm{GHz}$ with respect to the brightness peak at $8.4 \mathrm{GHz}$, our highest observing frequency (see Table 1). These shifts are produced by Synchrotron SelfAbsorption (SSA) of the jet plasma associated with the strong magnetic fields and particle density at the jet base. Taking into account the shifts for all the frequency pairs, we have determined the normalized linear core-shift [5], assuming a smooth compact jet with equipartition between particles and fields: $\Omega \sim 0.031$ parsec per GHz. Knowing the galaxy inclination $(\sim 14 \mathrm{deg})$ and the jet opening angle ( $\sim$ deg), Martí-Vidal et al. (2011)[11] derived an 

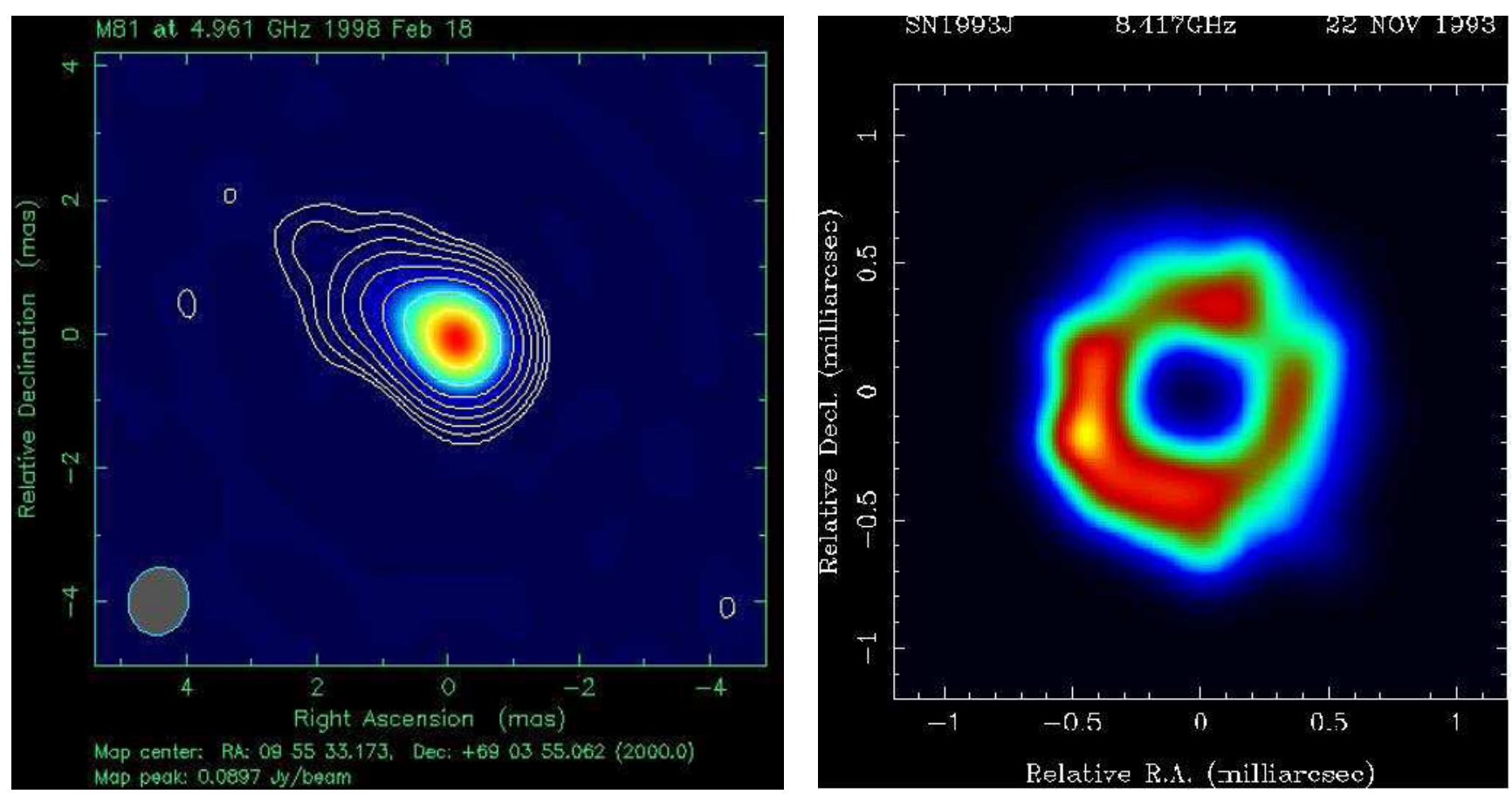

Figure 1. Left: VLBI Image of the low luminosity AGN at the heart of M81 at $5 \mathrm{GHz}$; Right: VLBI image of SN 1993J at 8.4 GHz at the time of the discovery of the shell-like structure (age: 239 days)[7]. At the distance of M81, 1 milliarcsec corresponds to $18 \mathrm{mpc}$.

Table 1. Time average shifts in the M81* brightness peak at 1.7, 2.3 and 5.0 GHz with respect to the $8.4 \mathrm{GHz}$ peak, taking the estimated center of the SN 1993J radio shell at each epoch as reference position.

\begin{tabular}{l|ccc}
\hline Frequency Pair (GHz) & $1.7-8.4$ & $2.3-8.4$ & $5.0-8.4$ \\
\hline Shift (mas) & $0.74 \pm 0.19$ & $0.59 \pm 0.09$ & $0.18 \pm 0.13$ \\
\hline
\end{tabular}

equipartition magnetic field at the jet base of the order of $10-50 \mathrm{mG}$. However, this determination is not consistent with the field required to model the inverted spectral index of M81*. On the other hand, assuming a magnetized black hole scenario, we have determined a mass for the central black hole of $\sim 2 \times 10^{7} \mathrm{M}_{\odot}$, which is in good agreement with those derived from the kinematics of the central disc of gas and from the stellar velocity dispersion at the bulge.

\section{Precession in the jet of M81*}

As a second step in the data analysis, we fitted a simple model to the M81 structure, consisting of an elliptical Gaussian, to model the core emission, and a circular Gaussian to model the jet extension. We analyzed both the astrometric and the model-fitting results in a consistent scheme.

In Fig. 2 we show the position of the brightness peak of M81 for different frequencies (shown with different symbols) and for different epochs (shown with different colors) resulting from the phase-referencing observations with respect to SN 1993J. The data suggest a systematic change in the orientation of the jet with time.

In Fig. 3 we show the model-fitting results: position angle of the major axis of the $5 \mathrm{GHz}$ core ellipse model (left) and the total VLBI correlated flux density for all frequencies (right) as a function of time. The most noticeable

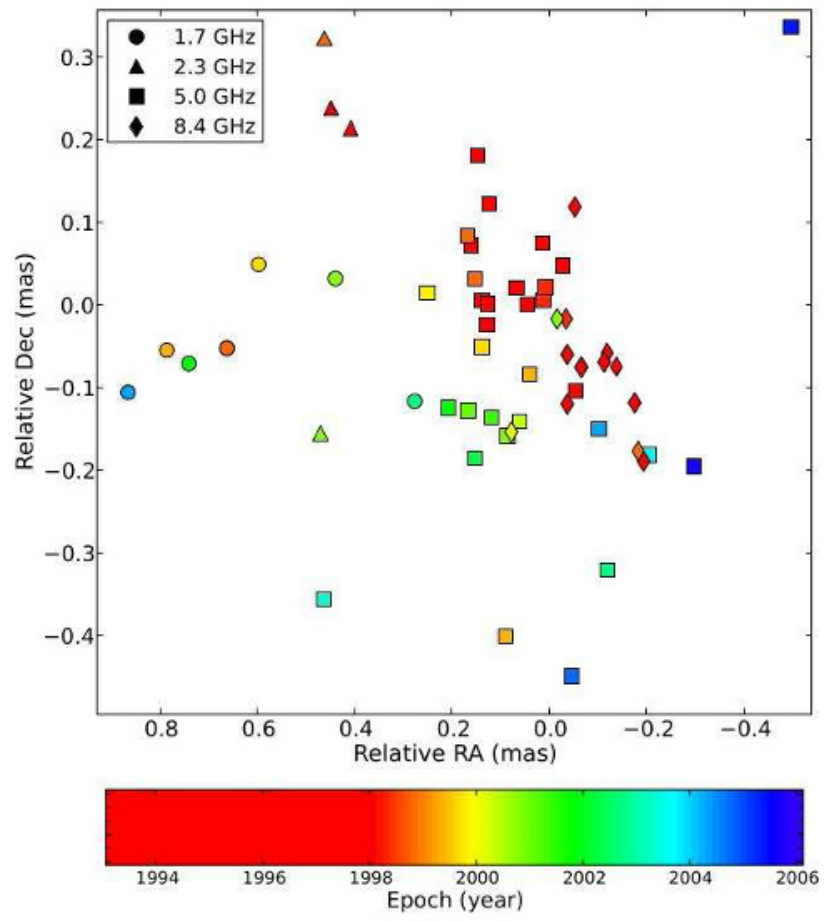

Figure 2. Absolute position of the VLBI brightness peak of the low luminosity AGN of M81; colors indicate time evolution, while symbols indicate frequency.[11] 

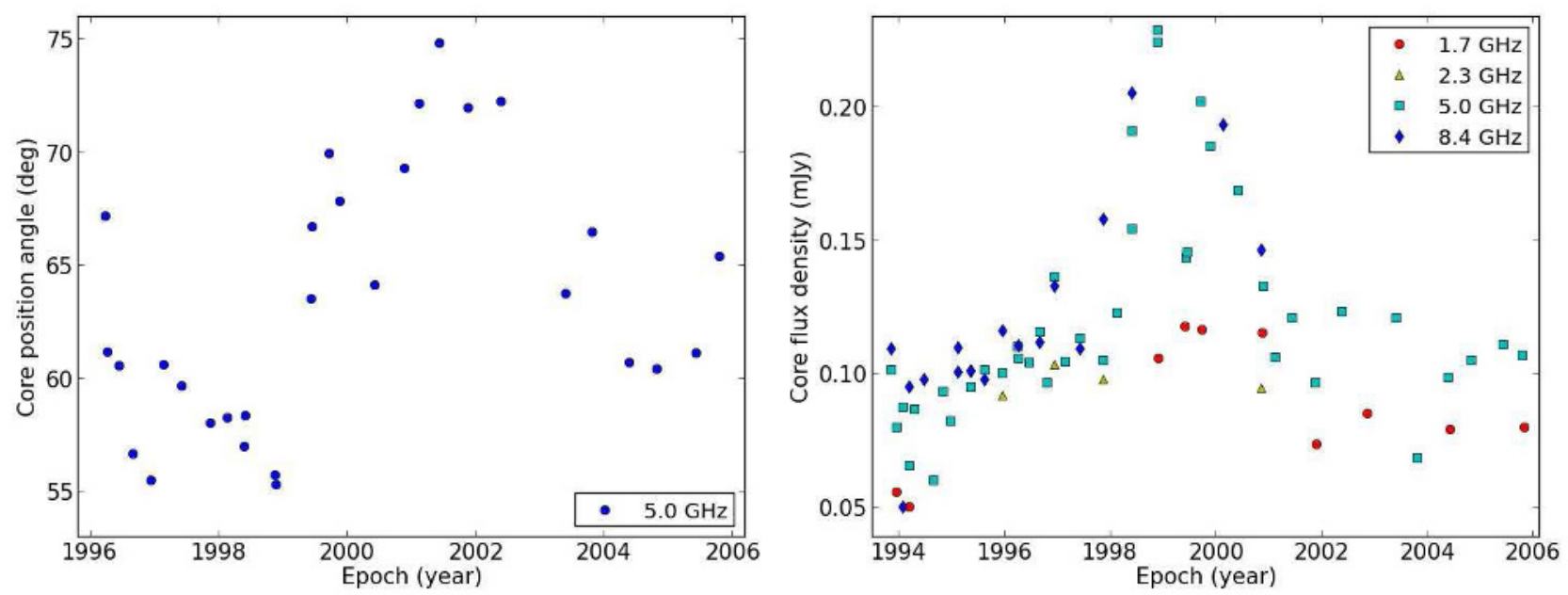

Figure 3. Left: Position Angle of the core of the LLAGN in M81 at $5 \mathrm{GHz}$ versus time; Right: Lightcurve of the core of the LLAGN in M81, obtained from our VLBI images, spanning the period 1994-2006.

result is the long flare that lasted about 4 years, between 1998 and 2002. This flare occurred on a time range where the position angles of the cores at all frequencies (referred to a fiducial point on the sky) increased sistematically; that is, the source turns to an East-West orientation. Simultaneously, the absolute position of the brightness peaks moves towards East, suggesting that it is the whole jet that turns Eastwards at the time the flare is happening.

This long flare in the radio emission of M81* seems to be directly related to changes in the source geometry. In fact, all observational results favour an scenario of jet precession. Another interesting result is that the variability during the long flare was higher at higher frequencies $(5 \mathrm{GHz}$ and $8.4 \mathrm{GHz}$ ) than at lower ones $(1.6 \mathrm{GHz})$, possibly due to the Doppler boosting effects associated with the jet bending. In fact, the observed range of changes in the Position Angle and the flux density variability can be explained by Doppler boosting effects, assuming a Lorentz factor $\gamma=10-20$ and deprojected variations of 2-4 degrees with respect to the "average" ridge line viewing angle (between 12 and 16 degrees, assuming $\theta_{\text {LOS }}=14$ ). Thus, a jet bending of just a few degrees along the first $\sim 100 \mathrm{mpc}$ of the relativistic jet of $\mathrm{M} 81^{*}$ can explain our results.

Our results suggest a periodic evolving jet orientation, consistent with a precessing jet. In fact, the results can be modelled by a simple sine-like model (with a period of $7.27 \pm 0.08$ years) together with a long-term component in order to account for a long drift towards the East. In the case of this periodic precession, a new flare should have happened between 2005 and 2008. Unfortunately, no VLBI observations are available from 2006 until 2010. However, starting around 2010, we have new VLBI observations of M81* phase-referenced to the supernova SN 2008iz in M82. In Fig.4 we show how the new core position angles fit the predictions of the model reported by Martí-Vidal et al. (2011).
Recent results from the MOJAVE survey (Lister et al. (2013)) show a number of jets $(\sim 12)$ displaying oscillatory trends, with fitted periods of 5 to 12 years. Since these periods are shorter than those estimated for the typical precession timescales of the system "accretion disk + black hole", these authors favour a model in which these sinusoidal trends correspond to lit-up portions of thin-ribbon like structures embedded within a broader conical outflow. Perucho et al. (2012) proposed the generation of these ribbon-like structures from Kelvin-Helmholtz pressure maxima within the jet.

\subsection{Future Prospects}

We are monitoring the jet of M81* at higher frequencies (43 and $86 \mathrm{GHz}$ ) with the VLBA in order to sample the relativistic jet with a linear resolution of $\sim 1-2 \mathrm{mpc}$, which corresponds to sizes of 100-300 Schwarzschild Radii. At these frequencies, the flux density variability should be stronger and the structural variability should be better traced. In fact, at $15 \mathrm{GHz}$ McHardy et al. (private communication) have detected flux-density variability as large as $200 \%$, with a rich substructure in the light curve. This overall flux density variability shoul be reflected in structural variability in the innermost regions of the jet. The observations are performed quasi-simultaneously, taking advantage of the "fast frequency switching" capabilities of the VLBA.

\section{Acknowledgements}

This research has been partially supported by the projects AYA2009-13036-CO2-01, AYA2009-13036-CO2-02, AYA2012-38491-CO2-01 and AYA2012-38491-CO202, this last one cofunded with FEDER funds. We acknowledge also partial support from the grant PROMETEO/2009/104 of the Generalitat Valenciana and 


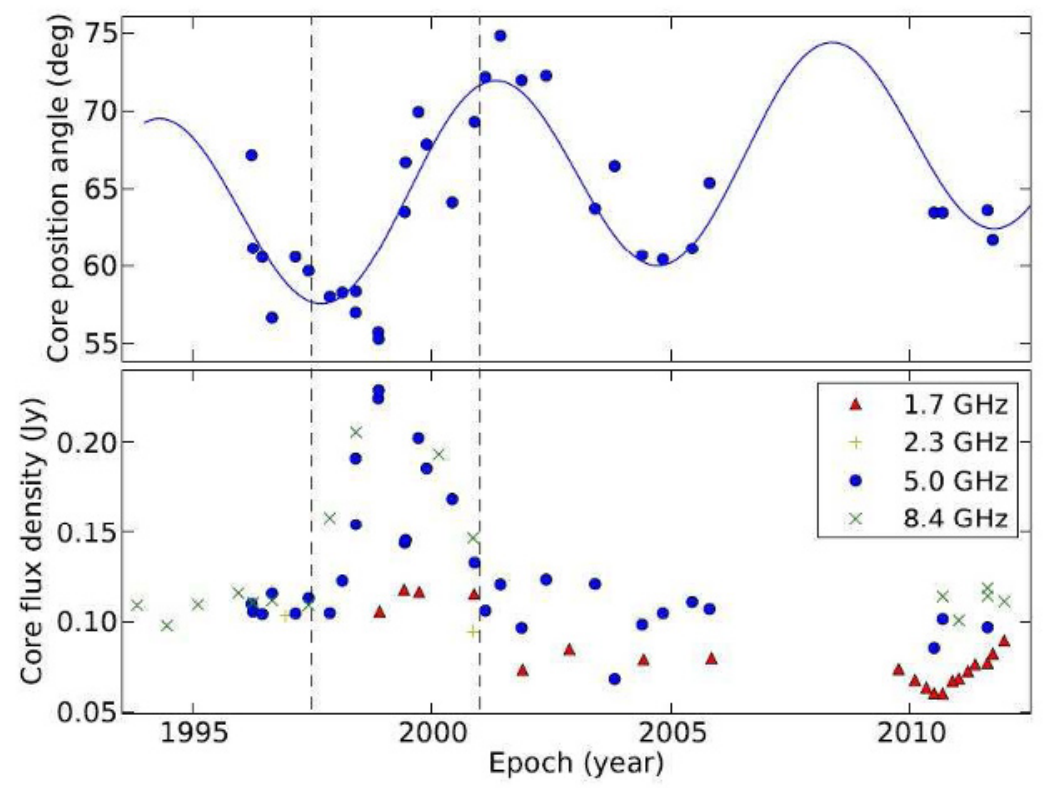

Figure 4. Top: Position Angle of the core of the LLAGN in M81 at $5 \mathrm{GHz}$ versus time, including data phase-referenced to SN 1993J (1994-2006) and to SN 2008iz in M82 (2010-2012). The sinusoidal precession model derived from the data in the observing period 1994-2006 is overplotted; Botttom: Lightcurve of the core of the LLAGN in M81, obtained from our VLBI images, spanning the period 1994-2012 (1994-2006: M81* phase-referenced to SN 1993J in M81; 2010-2012: M81* phase-referenced to SN 2008iz in M82).[? ]

the COST MP0905 Action "Black Holes in a Violent Universe".

\section{References}

[1] Devereux, N., Ford, H., Tsvetanov, Z., Jacoby, G., AJ, 125, 1226 (2003)

[2] Freedman, W.L., Hughes, S.M., Madore, B.F., et al., ApJ 427, 628 (1994)

[3] Ho, L.C., van Dyk, S.D., Pooley, G.G. et al., AJ, 118, 843 (1999)

[4] Lister, M. L., Aller, M. F., Aller, H. D. et al., 2013arXiv1308.2713L (2013)

[5] Lobanov, A., A\&A, 330, 79 (1998)

[6] Marcaide, J.M., Shapiro, I.I., ApJ, 276, 56 (1984)

[7] Marcaide, J.M., Alberdi, A., Ros, E. et al., Nature, 373, 44 (1995)
[8] Marcaide, J.M., Martí-Vidal, I., Alberdi, A. et al., A\&A, 505, 927 (2009)

[9] Martí-Vidal, I., Marcaide, J.M., Alberdi, A. et al., A\&A, 526, A142 (2011)

[10] Martí-Vidal, I., Marcaide, J.M., Alberdi, A. et al., A\&A, 526, A143 (2011)

[11] Martí-Vidal, I., Marcaide, J.M., Alberdi, A. et al., A\&A, 533, A111 (2011)

[12] Martí-Vidal, I., Marcaide, J.M., Alberdi, A., Brunthaler, A., POS (11th EVN Symposium) 065, arXiv:1301.4782 (2013)

[13] Perucho, M., Kovalev, Y. Y., Lobanov, A. P., Hardee, P. E., \& Agudo, I. ApJ, 749, 55 (2012)

[14] Reynolds, C.S., Nowak, M., Markoff, S. et al., ApJ, 691, 1159 (2009)

[15] Ros, E., Pérez-Torres, E., A\&A, 537, A93 (2012) 